\title{
ON THE MOTION OF A PENDULUM IN A TURBULENT FLUID*
}

\author{
BY \\ C. C. LIN \\ Guggenheim Laboratory, California Institute of Technology
}

1. Introduction. In a recent paper, Schumann ${ }^{1}$ has investigated the motion of a damped pendulum in a turbulent fluid by considering the effect of the fluid as a continuous fluctuating force. He first considers a damped pendulum which is bombarded by pellets of equal mass $m h$ at equal intervals $h$ of the time, and then treats the case of continuous fluctuations by a limiting process. For this latter case, which "must be regarded as being of far more practical importance," Schumann obtains the very interesting result: ${ }^{2}$

$$
\begin{gathered}
r(\xi)=\frac{1}{2 \int_{0}^{\infty} R(x) e^{-\lambda x} \sin (\beta x+\gamma) d x}\left[\int_{0}^{\infty}\{R(x+\xi)+R(x-\xi)\} e^{-\lambda x} \sin (\beta x+\gamma) d x\right. \\
\left.\quad+\int_{0}^{\xi}\{R(\xi-x)-R(x-\xi)\} e^{-\lambda x} \sin (\beta x+\gamma) d x\right] .
\end{gathered}
$$

The notation is as follows:

$r(\xi)=$ correlation function of the displacements of the pendulum at two instants separated by a time interval $\xi$;

$R(\xi)=$ limiting correlation function of the velocities of the impinging pellets;

$\lambda=l+m / M, M$ being the mass of the pendulum and $l$ its damping factor;

$\beta^{2}=\alpha^{2}+l^{2}-\lambda^{2}, 2 \pi / \alpha$ being the (damped) period of the pendulum; $\sin \gamma=\beta / \beta_{1}$

$\beta_{1}^{2}=\beta^{2}+\lambda^{2}=\alpha^{2}+l^{2}$.

The analysis used by Schumann is very elegant, but somewhat lengthy. In this article, we shall study the problem from another point of view, and give an alternative derivation of (1.1). This derivation, though unable to cover the case of discontinuous impacts, seems to show the nature of that relation much more clearly.

2. Damped pendulum under the action of a fluctuating force. We shall now investigate the correlation of displacement of a damped pendulum in relation to that of the exciting force. The notation used in this section should first be regarded as having different (though analogous) interpretations from

* Received Dec. 4, 1942.

${ }^{1}$ Schumann, T. E. W., Phil. Mag. (7), 33, 138-150 (1942).

${ }^{2}$ Loc. cit., eq. (57), p. 146. 
those used in $\$ 1$. The identification of the two systems of notation will be made in $\$ 3$.

Consider the equation of motion of the pendulum

$$
\frac{d^{2} y}{d t^{2}}+2 \lambda \frac{d y}{d t}+\left(\beta^{2}+\lambda^{2}\right) y=P,
$$

where $y$ is the displacement, $\lambda$ is the damping factor and $2 \pi / \beta$ is the (damped) period of the pendulum, $P$ is the exciting force per unit mass of the pendulum, and $t$ is the time. If the force $P$ is quasi-periodic, and is given by the real part of

$$
P=\sum A_{n} e^{i \omega_{n} t},
$$

where $\omega_{n}$ and $A_{n}$ are real and complex constants respectively, the steadystate displacement of the frequency $\omega_{n} / 2 \pi$ is given by the real part of

$$
y_{n}=a_{n} e^{i \omega_{n} t}, \quad a_{n}=\frac{A_{n}}{\left(\beta^{2}+\lambda^{2}-\omega_{n}^{2}\right)+2 i \lambda \omega_{n}} .
$$

Thus, we have

$$
\left|a_{n}\right|^{2}=\frac{\left|A_{n}\right|^{2}}{\left(\beta^{2}+\lambda^{2}-\omega_{n}^{2}\right)^{2}+4 \lambda^{2} \omega_{n}^{2}} .
$$

This is the relation between the spectrum of the displacement and that of the force in the case of discrete spectra. It is not difficult to generalize this result to the case of continuous spectra by the considerations of generalized harmonic analysis. ${ }^{3}$ We have then

$$
f(\omega)=A \frac{F(\omega)}{\left(\beta^{2}+\lambda^{2}-\omega^{2}\right)^{2}+4 \lambda^{2} \omega^{2}},
$$

where $f(\omega)$ and $F(\omega)$ are the spectra of the displacement and the force respectively,

$$
\int_{0}^{\infty} f(\omega) d \omega=1, \quad \int_{0}^{\infty} F(\omega) d \omega=1,
$$

and $A$ is a constant of normalization,

$$
\frac{1}{A}=\int_{0}^{\infty} \frac{F(\omega) d \omega}{\left(\beta^{2}+\lambda^{2}-\omega^{2}\right)^{2}+4 \lambda^{2} \omega^{2}} .
$$

These are the well-known relations in the phenomena of resonance.

The correlations $r(\xi)$ and $R(\xi)$ of the displacement and the force respectively stand in Fourier transform relations to the spectra4 (apart from conventional numerical factors):

3 Wiener, N., "The Fourier Integral" (Cambridge, 1933), p. 150.

- Wiener, N., loc. cit., eq. (21.21), p. 161, and discussions on p. 163. To be exact, we should follow Wiener in calling $f(\omega)$ and $F(\omega)$ the spectral densities. 


$$
\begin{array}{ll}
f(\omega)=\frac{2}{\pi} \int_{0}^{\infty} r(x) \cos \omega x d x, & r(\xi)=\int_{0}^{\infty} f(\omega) \cos \omega \xi d \omega \\
F(\omega)=\frac{2}{\pi} \int_{0}^{\infty} R(x) \cos \omega x d x, & R(\xi)=\int_{0}^{\infty} F(\omega) \cos \omega \xi d \omega .
\end{array}
$$

From (2.5), (2.8), and (2.9), we have at once

$$
r(\xi)=\frac{2 A}{\pi} \int_{0}^{\infty} \frac{\cos \omega \xi d \omega}{\left(\beta^{2}+\lambda^{2}-\omega^{2}\right)^{2}+4 \lambda^{2} \omega^{2}} \int_{0}^{\infty} R(x) \cos \omega x d x .
$$

It is not difficult to justify a change of the order of in tegration, since the correlation functions are expected to go to zero at infinity sufficiently rapidly. The above relation then becomes

$$
r(\xi)=\frac{2 A}{\pi} \int_{0}^{\infty} R(x) d x \int_{0}^{\infty} \frac{\cos \omega \xi \cos \omega x}{\left(\beta^{2}+\lambda^{2}-\omega^{2}\right)^{2}+4 \lambda^{2} \omega^{2}} d \omega .
$$

Since we have

$$
\cos \omega \xi \cos \omega x=\frac{1}{2}\{\cos \omega(\xi+x)+\cos \omega(\xi-x)\},
$$

we can evaluate the integral with respect to $\xi$ in $(2.11)$, if we know

$$
I(t)=\int_{0}^{\infty} \frac{\cos \omega t}{\left(\beta^{2}+\lambda^{2}-\omega^{2}\right)^{2}+4 \lambda^{2} \omega^{2}} d \omega .
$$

This integral is relatively easy to evaluate. We write

$$
I(t)=\frac{1}{2} \int_{-\infty}^{\infty} \frac{e^{i \omega t}}{\left(\beta^{2}+\lambda^{2}-\omega^{2}\right)^{2}+4 \lambda^{2} \omega^{2}} d \omega,
$$

and consider the corresponding contour integral in the complex $\omega$-plane, the contour being the usual one composed of the real axis and a semi-circle at infinity. The circle is taken in the upper half-plane if $t>0$, and in the lower half-plane if $t<0$. Since (2.13) shows that $I(t)$ is an even function, we shall carry out the calculations for $t>0$ alone.

There is no difficulty in showing that the integral over the semi-circle goes to zero. For, when the imaginary part of $\omega t$ is positive, $\left|e^{i \omega t}\right|$ is bounded, and $\left|\left(\beta^{2}+\lambda^{2}-\omega^{2}\right)^{2}+4 \lambda^{2} \omega^{2}\right|=O\left(|\omega|^{4}\right)$ for large values of $|\omega|$. The evaluation of (2.14) then reduces to the calculation of the residues of the integrand at the two simple poles $\pm \beta+i \lambda(\lambda>0)$ inside the contour. The result can be easily verified to be 


$$
I(t)=\frac{\pi e^{-\lambda t}}{4 \beta \lambda} \sin (\beta t+\gamma)
$$

where

$$
\sin \gamma=\frac{\beta}{\left(\beta^{2}+\lambda^{2}\right)^{1 / 2}} .
$$

With the help of (2.12), (2.13), and (2.15), the equation (2.11) becomes

$$
\begin{aligned}
r(\xi)= & \frac{A}{4 \beta \lambda}\left[\int_{0}^{\infty}\{R(x+\xi)+R(x-\xi)\} e^{-\lambda x} \sin (\beta x+\gamma) d x\right. \\
& \left.+\int_{0}^{\xi}\{R(\xi-x)-R(x-\xi)\} e^{-\lambda x} \sin (\beta x+\gamma) d x\right] .
\end{aligned}
$$

This is Schumann's relation (1.1), if the constants can be identified. There is no difficulty with the normalization coefficient. We have

$$
\begin{aligned}
\frac{4 \beta \lambda}{A} & =2 \int_{0}^{\infty} R(x) e^{-\lambda x} \sin (\beta x+\gamma) d x \\
& =4 \beta \lambda \int_{0}^{\infty} \frac{F(\omega) d \omega}{\left(\beta^{2}+\lambda^{2}-\omega^{2}\right)^{2}+4 \lambda^{2} \omega^{2}},
\end{aligned}
$$

on putting $\xi=0$ in (2.17) and recalling (2.7). The second relation is a byproduct of our investigation. The limiting case $\lambda \rightarrow 0$ reduces to the wellknown relation (2.9).

Referring to (2.9), we see that $R(\xi)$ is an even function of $\xi$, so that the second integral in (2.17) may be dropped.

From the derivation, we see that (2.17) is nothing but the Fourier transform of the well-known resonance relation (2.5).

3. Identification of the results. We shall now identify Schumann's result with ours by showing that in the limiting case, his pendulum has an effective damping factor $\lambda$ instead of $l$, and that his correlation of velocity of the pellets becomes the correlation of force. The equations of motion as given by Schumann are ${ }^{5}$

$$
u_{r}=\frac{M-m h}{M+m h} u_{r}^{\prime}+\frac{2 m h}{M+m h} v_{r},
$$

at the $r$ th impact at the instant $t=r h$, and

$$
\frac{d^{2} y}{d t^{2}}+2 l \frac{d y}{d t}+\left(\alpha^{2}+l^{2}\right) y=0,
$$

${ }^{5}$ Loc. cit., eqs. (1), (2). It seems that there are some misprints in the original paper. 
between successive impacts. In (3.1), $u_{r}^{\prime}$ and $u_{r}$ denote respectively the velocities of the mass $M$ just before and just after the $r$ th impact, and $v_{r}$ is the velocity of the $r$ th pellet.

In the limiting case, the discrete impacts become a continuous force given by

$$
P_{1}=\lim _{h \rightarrow 0} \frac{u_{r}-u_{r}^{\prime}}{h} M=-2 m u+2 m v
$$

where $u$ and $v$ are the limiting values for $u_{r}$ and $v_{r}$ at the instant $t=r h$. Thus $u$ is evidently the velocity of the pendulum $d y / d t$, and $v$ is the velocity of the infinitesimal impinging pellet in the interval $(t, t+d t)$. The equation of motion (3.2) then becomes

$$
\frac{d^{2} y}{d t^{2}}+2 l \frac{d y}{d t}+\left(\alpha^{2}+l^{2}\right) y=\frac{1}{M}\left(-2 m \frac{d y}{d t}+2 m v\right) .
$$

We see that this limiting case carries an inherent damping factor $m / M$ in addition to the damping factor $l$. The term $2 m v / M$ is evidently the exciting force per unit mass, since $m$ is the impinging mass per second, the factor 2 corresponding to the fact that when the impinging mass is infinitesimal compared with the colliding mass, the former rebounds with the colliding speed,a fact of ten used in the kinetic theory of gases.

The identification of Schumann's result with that given in $\$ 2$ is therefore complete.

4. Discussion. In view of the above derivation, we must be a little careful in applying Schumann's relation (1.1) to the study of the motion of a pendulum in a turbulent fluid. The correlation function $R$ obtained (by suitable processes) according to that relation from the correlation function $r$ of the displacements is that for the hydrodynamic force (the part corresponding to an extra damping being removed). In a turbulent fluid, the connection between the velocity fluctuations of the fluid before the introduction of a pendulum and the fluctuating force acting upon the pendulum after its introduction is not at once evident. A preliminary careful investigation seems to be necessary before the method suggested by Schumann could be used with advantage.

It is also clear that the spectrum of velocity fluctuations of the pendulum will be proportional to $\omega^{2} f(\omega)$. It is then clear from (2.8) that the correlation function of the velocity fluctuations will be proportional to $-r^{\prime \prime}(\xi)$.

There is another point which should be mentioned. The spectrum and correlation function discussed above refer to those observed at a fixed spatial point, if we assume the pendulum to be never far from its position of equilibrium. It is not difficult to observe this spectrum with a hot-wire anemome- 
ter, as has been done by several observers. ${ }^{6}$ The Fourier transform of this spectrum will then give the correlation of the fluctuations at successive instants at the same point in space. It is not clear, however, how (as Schumann suggested) the pendulum can be used to observe the correlation function defined with respect to the same material point,- the quantity introduced by Taylor $^{7}$ in the Lagrangian description of turbulence for the study of the phenomenon of turbulent diffusion. ${ }^{8}$

In conclusion, the author wishes to express his sincere thanks to Professor Theodore von Kármán for suggesting the problem to him and for his invaluable suggestions.

${ }^{6}$ For example, L. F. G. Simmons and C. Salter, Proc. Roy. Soc. Ser. A, 165, 73-89 (1938); H. L. Dryden, Proceedings of the Fifth International Congress of Applied Mechanics (Cambridge, Mass., 1938), pp. 362-367; H. Motzfeld, Zeits. f. angew. Math. u. Mech. 18, 362-365 (1938).

7 Taylor, G. I., Proc. Lond. Math. Soc., (2), 20, 196-212, (1921). Clearer statements regarding this point are made in his paper of 1935, Proc. Roy. Soc., A, 151, 421-478 (1935).

8 A discussion of the three types of correlations that may be defined in the study of isotropic turbulence has been made by G. Dedebant and P. Wehrle, Comptes Rendus 208, 625628 (1939). 